\title{
A NEW CLASS OF COMPOSITION OPERATORS
}

\section{S. PATTANAYAK, C.K. MOHAPATRA and A.K. Mishra}

\author{
School of Mathematical Sciences \\ Sambalpur University \\ Jyot1 Vihar 768019, INDIA
}

(Received May 30, 1984 and in revised form June 14, 1985)

ABSTRACT. A new class of composition operators $P_{\phi}: H^{2}(T) \rightarrow H^{2}(T)$, with $\phi: T \rightarrow \bar{D}$ is introduced. Sufficient conditions on $\phi$ for $P_{\phi}$ to be bounded and Hilbert-Schmidt are obtained. Properties of $P_{\phi}$ with $\phi\left(e^{1 t}\right)=a e^{i t}+b e^{-1 t}$ for different values of the parameters $a$ and $b$ have been investigated. This paper concludes with a discussion on the compactness of $P_{\phi}$.

KEY WORDS AND PHRASES. IP Space, Composition operator, Hilbert Schmidt operator, Compact operator.

1980 AMS SUBJECT CLASSIFICATION CODES. 47B37, 47B99, $46 J 15$.

1. PRELIMINARIES.

For a complex valued function $f$ analytic in $D=\{z:|z|<1\}$ and for $1 \leq p \leq \infty$ set

$$
M_{p}(r, f)=\left(f_{0}^{2 \pi}\left|f\left(r e^{i \theta}\right)\right|^{p} \frac{d \theta}{2 \pi}\right)^{\frac{1}{p}}, \quad 1 \leq p<\infty
$$

and

$$
M(r, f)=\operatorname{Sup}_{0<\theta<2 \pi}\left|f\left(r e^{1 \theta}\right)\right| .
$$

The function $f$ is said to be in $H^{P}(D)$, if $\underset{r \rightarrow 1-}{\lim } M_{p}(r, f)<\infty$. Similarly, let $H^{P}(T), T=\{z:|z|=1\}$, be the class of functions in ${ }^{P}(T)$ such that

$$
\int_{0}^{2 \pi} f\left(e^{i \theta}\right) e^{i n \theta} d \theta=0, n=1,2,3 \ldots \ldots .
$$

It is known $[1,2]$ that for $f$ in $H^{P}(D) \underset{r i 1-}{1 \text { im }} f\left(r e^{1 \theta}\right)=f_{*}\left(e^{i \theta}\right)$ exists for almost all $\theta$ and $f_{*}$ belongs to $H^{P}(T)$. Conversely, the Poisson integral of a function in $H^{P}(T)$ is in $H^{P}(D)$. Also, if $f$ in $H^{P}(D)$ has the sequence $\left\{a_{n}\right\}$ as its Taylor coefficients then, $f_{*}$ has the same sequence as its Fourier coefficients and vice versa. This correspondence establishes an isometrical isomorphism between $H^{p}(D)$ and $H^{p}(T)$. Thus, these 
two spaces are interchangeably used and are usually referred to as the Hardy Space ${ }_{\mathrm{H}}^{\mathrm{P}}[1,2]$.

In the sequel we came across another space familiarly known as the weighted Hardy space [3]. Let $\rho(n)$ be a sequence of positive numbers. An analytic function $f: D \rightarrow C$, given by $f(z)=\sum a_{n} z^{n}$, is said to be in the class $H^{2}(\rho)$, if $\left.|| f\right|_{\rho}=\Sigma\left|a_{n}\right|^{2} \rho(n)<\infty$. Also we need the following definition. Let $\mathrm{H}$ be a Hilbert space and $\mathrm{T}$ be a bounded linear operator on $\mathrm{H}$. Then, $\mathrm{T}$ is sald to be Hilbert Schmidt if there exists an orthonormal basis $\left\{\mathrm{e}_{\mathrm{n}}\right\}$ in $\mathrm{H}$ such that $\Sigma|| \mathrm{Te} \mathrm{n}_{\mathrm{n}} \|^{2}<\infty$.

Throughout in the present paper we denote by $e_{n}, n=0,1,2, \ldots$, the function $e_{n}\left(e^{i t}\right)=e^{i n t}$. We note that $\left\{e_{n}\right\}$ forms an orthonormal basis for $H^{2}$.

2. A NEW CLASS OF CONPOSITION OPERATORS.

Let $\phi: D \rightarrow D$ be analytic and let $C_{\phi}:{ }_{H}^{P}(D) \rightarrow{ }_{H}^{P}(D)$ be defined by $\left(C_{\phi} f\right)(z)=$ $\mathrm{f}(\phi(z)), z$ in $D$. The operator $\mathrm{C}_{\phi}$ is known as a composition operator on $\mathrm{H}^{\mathrm{p}}(\mathrm{D})$ and is extensively studied in the literature [4]. In the present paper we introduce and study a new class of composition operators $P_{\phi}$ on $H^{2}(T)$ where $\phi: T \rightarrow \bar{D}$ many be 'nonanalytic' also. That is $\phi$ nonvanishing negative Fourier coefficients.

DEFINITION. Let $\phi: \mathrm{T} \rightarrow \overline{\mathrm{D}}$ satisfy the following properties:

(a) for every set $E \subset T$, of linear measure zero, $\phi^{-1}(E)=\{z \varepsilon T$ : $\phi(z)=w, w \varepsilon T\}$ is also a set of linear measure zero and

(b) for every $f$ in $\mathrm{H}^{2}(\mathrm{~T})$, foф is in $\mathrm{L}^{2}(\mathrm{~T})$.

Then, define $P_{\phi}: H^{2}(T) \rightarrow H^{2}(T)$ by $P_{\phi} f=P(f \circ \phi)$ where $P$ is the projection of $L^{2}(T)$ into $\mathrm{H}^{2}(\mathrm{~T})$.

Here some explanations are in order. We observe that a function $f$ in $H^{2}(T)$ can be extended analytically into $D$ as described in Section 1 . So with the condition (a), fo $\phi$ is defined almost everywhere on $T$. Futher, let $f$ be represented by the Fourier series $\sum_{n=0}^{\infty} a_{n} e^{i n \Theta}$. Then by the Weierstrass theorem, $\sum_{n=0}^{\infty} a_{n}\left(\phi\left(e^{1 \theta}\right)\right)^{n}$ converges pointwise to $f\left(\phi\left(e^{1 \theta}\right)\right)$ for all $\theta$ such that $\phi\left(e^{1 \theta}\right) \varepsilon D$ and by a result of Carleson [5] $\sum^{\infty}=0 a_{n}\left(\phi\left(e^{i \theta}\right)\right)^{n}$ converges pointwise to $f\left(\phi\left(e^{i \theta}\right)\right)$ for almost all $\theta$ such that $\phi\left(e^{i \theta}\right) \varepsilon T$. Hence $\sum_{n=0}^{\infty} a_{n}\left(\phi\left(e^{i \theta}\right)\right)^{n}$ converges pointwise almost everywhere on $T$ to $f\left(\phi\left(e^{i \theta}\right)\right)$. Thus throughout in this paper we write $\sum_{\sum_{0}^{\infty}}^{\infty} a_{n}\left(\phi\left(e^{1 \theta}\right)\right)^{n}$ in place of $f\left(\phi\left(e^{1 \theta}\right)\right)$.

We note theat if $\phi$ satisfies the conditions of the definition, then by the Closed Graph Theorem, $P_{\phi}$ is a bounded operator. So a natural question is: under what conditions on $\phi$, fo $\phi$ is in $\mathrm{L}^{2}(\mathrm{~T})$ for all $\mathrm{f}$ in $\mathrm{H}^{2}(\mathrm{~T})$. The present paper primarily deals with this question.

In the following sections we first obtain bounds for the norm of $P_{\phi}$ under suitable conditions on $\phi$. Then we consider $\phi$ defined by $\phi\left(e^{i t}\right)=a e^{i t}+b e^{-i t}$ and study 
conditions on $a$ and $b$ such that fo $\varepsilon \varepsilon L^{2}(T)$ for all $f$ in $H^{2}(T)$. In the last section we have discussed the compactness of $P_{\phi}$ with the help of some examples.

3. NORM OF $\mathrm{P}_{\phi}$.

We have the following results.

THEOREM 1. Let $\phi: T \rightarrow \bar{D}$ be such that

$$
\int_{0}^{2 \pi} \frac{d t}{1-\left|\phi\left(e^{i t}\right)\right|^{2}}=M(\phi)<\infty
$$

Then, $\mathrm{P}_{\phi}$ is Hilbert Schmidt and || $\mathrm{P}_{\phi} \| \leq(\mathrm{M}(\phi) / 2 \pi)^{1 / 2}$

PROOF. Let $f$ in $H^{2}(T)$ be given by $f(z)=\sum a_{n} z^{n}, z$ in $T$. Then,

$$
\begin{aligned}
\mid f\left(\left.\phi\left(e^{i \theta}\right)\right|^{2}\right. & =\left|\sum a_{n}\left(\phi\left(e^{1 \theta}\right)\right)^{n}\right|^{2} \leq\left(\Sigma\left|a_{n}\right|^{2}\right)\left(\Sigma\left|\phi\left(e^{1 \theta}\right)\right|^{2 n}\right) \\
& =\left.|| f\right|^{2} \frac{1}{1-\left|\phi\left(e^{1 \theta}\right)\right|^{2}} \quad \text { a.e. }
\end{aligned}
$$

So,

$$
\left\|P_{\phi} f\right\|^{2} \leq\|f \circ \phi\|_{2}^{2} \leq\|f\|_{2}^{2} \frac{M(\phi)}{2 \pi}
$$

and we get ||$P_{\phi}|| \leq(M(\phi) / 2 \pi)^{1 / 2}$.

Next, with the orthonormal basis $e_{n}, n=0,1,2, \ldots$ of $H^{2}(T)$, we have

$$
\stackrel{\sum}{=0}_{0}^{\infty} \| P_{\phi}\left(e_{r}\right)||^{2} \leq \sum_{n=0}^{\infty}|| e_{n} o \phi||^{2}=\sum_{n=0}^{\infty} \frac{1}{2 \pi} \int_{0}^{2 \pi}\left|\phi\left(e^{i t}\right)\right|^{2 n} d t=(M(\phi) / 2 \pi)<\infty .
$$

Thus, $P_{\phi}$ is Hilbert Schmidt.

COROLLARY 2. If $\phi: T \rightarrow D$ is continuous then $P_{\phi}$ is Hilbert Schmidt.

PROOF. The condition (3.1) is trivially satisfied if $\phi$ is continuous.

By an example in the next section we will show that (3.1) is only a sufficient condition for $P_{\phi}$ to be Hilbert Schmidt. We need the following lemma due to Gabriel [6] for the proof of our next theorem.

LEMMA. Let $\Gamma$ be a rectifiable convex curve in the closed unit disc. Then, for every $f$ in $\mathrm{H}^{2}$

THEOREM 2. Let $\phi: T \rightarrow \bar{D}$ be such that

$$
\int_{\Gamma}|f(w)|^{2}|d w| \leq 4 \pi|| f||_{2}^{2}
$$

(i) $\phi$ describes a closed rectifiable convex curve in $\bar{D}$ and

(ii) $m=\operatorname{mff}\left|\phi^{\prime}\left(e^{i t}\right)\right|>0,0 \leq t \leq 2 \pi$,

Then, $\quad\left\|P_{\phi}\right\| \leq(2 / m)^{1 / 2}$.

PROOF. By lemma and the condition (ii) we have

$$
4 \pi|| f||_{2}^{2} \geq \int_{0}^{2 \pi}\left|f\left(\phi\left(e^{i t}\right)\right)\right|^{2}\left|\phi^{\prime}(t)\right| d t \geq m \int_{0}^{2 \pi}\left|(f \circ \phi)\left(e^{i t}\right)\right|^{2} d t
$$

so that

$$
\left\|P_{\phi} f\right\|^{2} \leq \frac{1}{2 \pi} \int_{0}^{2 \pi}\left|(f \circ \phi)\left(e^{i t}\right)\right|^{2} d t \leq \frac{2}{m}|| f||_{2}^{2}
$$


The conditions ( $i$ ) and (ii) in the above theorem are not necessary for $P_{\phi}$ to be bounded. As an example consider

$$
\phi(t)=\left(e^{i t}\right)=\left[\begin{array}{ll}
\mathrm{e}^{i t} & 0<t<\pi \\
0 & \pi \leq t \leq 2 \pi
\end{array}\right.
$$

so that $\phi$ does not satisfy any of the conditions (i) or (ii) of the theorem.

Now,

$$
\left.\frac{1}{2 \pi} \int_{0}^{2 \pi} \mid f(\phi)\left(e^{i t}\right)\right)\left.\right|^{2} d t=\frac{1}{2 \pi} \int_{0}^{\pi}\left|f\left(e^{i t}\right)\right|^{2} d t+\frac{1}{2 \pi} \int_{\pi}^{2 \pi}|f(0)|^{2} d t \leq|| f||_{2}^{2} .
$$

This shows that $P_{\phi}$ is bounded with $\left\|P_{\phi}\right\| \leq \sqrt{ } 2$.

4. A FAMILY OF COMPOSITION OPERATORS.

In this section we study the properties of $P_{\phi}$ for the particular family of functions $\phi: T \rightarrow \vec{D}$ given by

$$
\phi(z)=a z+b \bar{z}, \quad z \varepsilon T
$$

where $|a|+|b| \leq 1$. We note that if $|a| \neq|b|$ then the curve traced by $\phi$ is an ellipse containing the orgin in its interior. Also $m=$ inf $\left|a e^{-i t}-b\right| \geq|| a|-| b||>0$. Hence by Theorem 2, $P_{\phi}$ is bounded. It turns out that $P_{\phi}$ has many interesting properties for different values of the parameters $a$ and $b$. We need the following technical lemma.

LEMMA 1. For all $\mathrm{n}, \mathrm{k}$ in $\mathrm{z}_{+}$

$$
\left(\begin{array}{c}
n+2 k \\
k
\end{array}\right)^{+}<2^{n+2 k}
$$

PROOF. We shall prove (4.2) by method of induction on $n$. Let $n=0$ so that we have to show

$$
\left(\begin{array}{l}
2 k \\
k
\end{array}\right)<2^{2 k} \text { for } k=1,2,3, \ldots \ldots \ldots \text {. }
$$

We establish (4.3), also by the process of induction on $k$. For $k=1,\left(\begin{array}{l}2 \\ 1\end{array}\right)=2<2^{2}$ is trivially true. Next, assume that

$$
\left(\begin{array}{c}
2 k \\
k
\end{array}\right)<2^{2 k}, \text { i.e. } \frac{(2 k) !}{(k !)(k !)}<2^{2 k}
$$

To complete induction on $k$ we consider

$$
\left(\begin{array}{c}
2(k+1) \\
k+1
\end{array}\right)=\frac{(2 k+2) !}{(k+1) !(k+1) !}<2^{2 k} 2 \frac{(2 k+1)}{(k+1)}<2^{2(k+1)} .
$$

Thus, (4.3) is true for all $k=1,2,3 \ldots$. Next, let $n=1$. Then,

$$
\left(\begin{array}{c}
2 k+1 \\
k
\end{array}\right)=\left(\begin{array}{c}
2 k \\
k
\end{array}\right) \frac{(2 k+1)}{(k+1)}<2^{k} \cdot 2=2^{k+1} \text {. }
$$

Now, assume that $\left(\begin{array}{c}n+2 k \\ k\end{array}\right)<2^{n+2 k}$. To complete the induction we consider

$$
\left(\begin{array}{c}
n+1+2 k \\
k
\end{array}\right)=\frac{(n+2 k) !}{(k !)(n+k) !} \frac{(n+1+2 k)}{(n+1+k)}<2^{n+2 k} \cdot 2<2^{n+1+2 k} .
$$

Thus (4.3) is true for a11 $k=1,2, \ldots \ldots$ and $n=0,1,2 \ldots \ldots$. 
THEOREM 3. Let $\phi: T \rightarrow \bar{D}$ be given by $\phi(z)=a z+b \bar{z}, z$ in $T$.

(1) If $|a|+|b| \leq 1,|a| \neq|b|, b \neq 0$ and $|a|<\frac{1}{2}$ then $P_{\phi}$ is Hilbert Schmidt.

(ii) If $|a|+|b|=\frac{1}{2}$ then fo $\phi$ need not be in $L^{2}$ for all $f$ in $H^{2}$ so that $P_{\phi}$ is not defined on the whole of $\mathrm{H}^{2}$.

(iii) The inequality $|a|<\frac{1}{2}$ in (1) is best possible.

PROOF. Consider the orthonormal basis $e_{n}, n=0,1,2, \ldots$, for $H^{2}$. With respect to this basis $P_{\phi}$ has a matrix representation

where $m, n$, and $k \in z_{+} \cdot$ Now

$$
t_{m n}=\left[\begin{array}{ll}
0 & \text { if } n<m \\
\left(\begin{array}{l}
n+2 k \\
k
\end{array}\right) a^{n}(a b)^{k} & \text { if } n-m=2 k \\
0 & \text { if } n-m=2 k+1
\end{array}\right.
$$

$$
\begin{aligned}
& \left.\Sigma\left|t_{m, n}\right|^{2}=\sum_{n=0}^{\infty} \sum_{m=0}^{\infty} \quad \begin{array}{c}
n+2 k \\
k
\end{array}\right)^{2} \quad|a|^{2 n}|a b|^{2 k}
\end{aligned}
$$

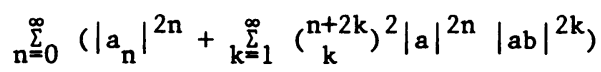

$$
\begin{aligned}
& =\frac{1}{1-|a|^{2}}+\sum_{n=0}^{\infty} k_{k=1}^{\infty}\left(\begin{array}{c}
n+2 k \\
k
\end{array}\right)^{2}|a|^{2 n}|a b|^{2 k}
\end{aligned}
$$

We use Lemma 1 to show that the second sum in the right hand side is convergent.

$$
\begin{aligned}
\mathrm{k}_{=1}^{\infty} \mathrm{n}_{\mathrm{n}=0}^{\infty}\left(\begin{array}{c}
\mathrm{n}+2 \mathrm{k} \\
\mathrm{k}
\end{array}\right)^{2}|\mathrm{a}|^{2 \mathrm{n}}|\mathrm{ab}|^{2 \mathrm{k}} & \leq \Sigma \Sigma 2^{2 \mathrm{n}+4 \mathrm{k}}|\mathrm{a}|^{2 \mathrm{n}}|\mathrm{ab}|^{2 \mathrm{n}}=\Sigma \Sigma|2 \mathrm{a}|^{2 \mathrm{n}}|4 \mathrm{ab}|^{2 \mathrm{k}} \\
& =\frac{1}{1-|2 \mathrm{a}|^{2}} \cdot \frac{1}{1-|4 \mathrm{ab}|^{2}}<\infty,
\end{aligned}
$$

since $|a|<\frac{1}{2}$, which also implies $|a b|<\frac{1}{4}$. This proves that $P_{\phi}$ is Hilbert

Schmidt. (11) For the proof of (11) consider the $\mathrm{H}^{2}$ function $f(z)=(1-z)^{-\alpha}, 0<\alpha<\frac{1}{2}$. For $a=b=\frac{1}{2}, \phi\left(e^{1 \theta}\right)=\cos \theta$. Thus,

$$
f\left(\phi^{\prime}\left(e^{1 \theta}\right)\right)=f(\cos \theta)=\frac{1}{(1-\cos \theta)^{\alpha}}=\frac{1}{\left(2^{\alpha} \sin ^{2 \alpha} \frac{\theta}{2}\right)} \quad \text { a.e. }
$$

and

$$
\int_{0}^{2 \pi} \mid\left(\left.f\left(\phi\left(e^{1 \theta}\right)\right)\right|^{2} d \theta=\int_{0}^{\pi / 2} \frac{2^{2-2 \alpha}}{\sin ^{4 \alpha} \theta} d \theta \geq \int_{0}^{\pi / 2} \frac{2^{2-2 \alpha}}{\theta^{4 \alpha}} d \theta=\infty\right.
$$

if $\alpha>\frac{1}{4}$. In the above we have made use of the well known inequality $\frac{2 \theta}{\pi}<\sin \theta<\theta$ for $0<\theta<\frac{\pi}{2}$.

(iii) In view of (ii), for the proof of (iii), it is sufficient to show that $P_{\phi}$ is not Hilbert Schmidt if $a+b=1$ and $a>b$. In fact, we show that under the above condition $\sum_{\mathrm{m}}\left|\mathrm{t}_{\mathrm{m}, \mathrm{m}}\right|^{2}=\infty$.

Observe that 


$$
e_{n}\left(\phi\left(e^{i \theta}\right)\right)=\left(a e^{i \theta}+b e^{-i \theta}\right)^{n}=\sum_{k=0}^{n}\left(\begin{array}{l}
n \\
k
\end{array}\right) a^{n-k} b^{k} e^{i(n-k) \theta} e^{-i k \theta} \text {. }
$$

Since $(a+b)^{n}=1$, we have $\sum_{k=0}^{n}\left(\begin{array}{l}n \\ k\end{array}\right) a^{n-k} b^{k}=1$. Hence considering this sum as the inner product of two vectors

$$
\left[\left(\begin{array}{c}
n \\
0
\end{array}\right) a^{n} b^{0},\left(\begin{array}{c}
n \\
1
\end{array}\right) a^{n-1} b^{1}, \ldots,\left(\begin{array}{c}
n \\
n
\end{array}\right) a^{0} b^{n} \quad \text { and } \mid 1,1 \ldots(n+1) \text { terms } 1\right. \text { ] }
$$

We see that, since $\|[1,1, \ldots, 1]\|^{2}=(n+1)$, by Cauchy Schwarz Inequality

$$
\sum_{k=0}^{n}\left|\left(\begin{array}{l}
n \\
k
\end{array}\right) a^{n-k} b^{k}\right|^{2} \geq \frac{1}{(n+1)} \text {. }
$$

Further, we observe that if $a>b$ then $\left(\begin{array}{l}n \\ r\end{array}\right) a^{n-r} b^{r}>\left(\begin{array}{c}n \\ n-r\end{array}\right) a^{r} b^{n-r}$ so that over half of the above sum is from terms where $n-k \geq k$ and so $\left\|P_{\phi} e_{n}\right\|^{2} \geq 1 / 2(n+1)$, leading us to $\sum_{n}\left|t_{n, n}\right|^{2}=\infty$. This completes the proof of the theorem.

Also, with the help of the same function $\phi(z)=a z+b \bar{z}$, we show that the condition (3.1) of Theorem 1 is not a necessary condition for $P_{\phi}$ to be a Hilbert Schmidt. For this take $a, b$ in $R, a b>0$ and $|a|+|b|=1$. Then,

$$
\int_{0}^{2 \pi} \frac{d t}{1-\left|\phi\left(e^{i t}\right)\right|^{2}}=\int_{0}^{2 \pi} \frac{d t}{\left(1-(a-b)^{2}\right) \sin ^{2} t}=\infty \text {. }
$$

However, in view of Theorem 3, it follows that $P_{\phi}$ is Hilbert Schmidt.

In the following theorem we present a sufficient condition on $f$ in $H^{2}$ to ensure that fo $\phi$ is in $L^{2}(T)$.

THEOREM 4. Let $\phi: T \rightarrow \bar{D}$ be given by $\phi(z)=a z+b \bar{z} \cdot|a|=|b|=\frac{1}{2}$ and $f$ in $H^{2}$ be given by $f(z)=\sum_{n=0}^{\infty} a_{n} z^{n}$. Further if $a_{n}=0\left(\frac{1}{n}\right)$ with $\alpha>\frac{3}{4}$, then fo $\phi \varepsilon L^{2}$. PROOF. First let $a=b=\frac{1}{2}$, so that $\phi\left(e^{1 \theta}\right)=\cos \theta$. Now,

$$
\left|f\left(\phi\left(e^{1 \theta}\right)\right)\right|^{2} \leq\left|\sum_{n=0}^{\infty} \frac{\cos ^{n} \theta}{n^{\alpha}}\right|^{2}
$$

We know that

$$
\frac{1}{(1-z)^{\beta+1}}=\sum_{n=0}^{\infty}\left(\begin{array}{c}
n+\beta \\
n
\end{array}\right) z^{n}
$$

and [7]

$$
\left(\begin{array}{c}
n+\beta \\
n
\end{array}\right) \sim \frac{n^{\beta}}{\Gamma(\beta+1)} .
$$

Taking $\beta=-\alpha$ in $(4.5)$ and $(4.6)$, we get

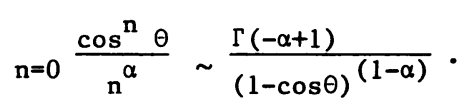

Thus, to complete the proof, it is sufficient to show that

$$
\int_{0}^{2 \pi}(1-\cos \theta)^{(2 \alpha-2)} \mathrm{d} \theta=\int_{0}^{2 \pi} \sin ^{(4 \alpha-4)} \frac{\theta}{2} \mathrm{~d} \theta<\infty .
$$

However, this is ture because of the condition $\alpha>\frac{3}{4}$. To dispose of the general case 
we observe that if $2 a=e^{1 \alpha}$ and $2 b=e^{1 \gamma}$ then $\phi\left(e^{1 \theta}\right)=e^{1(\alpha+\gamma) / 2} \cos \left(\frac{\alpha-\gamma+2 \theta}{2}\right)$ and this leads to simflar calculations as above.

Taking cue from the above theorem we next show that fo $\phi \varepsilon \mathrm{L}^{2}$ for $f \in \mathrm{H}^{2}(\rho(n))$ for a suitable choice of the sequence $\rho(n)$.

THEOREM 5. Let $\phi: T \rightarrow \bar{D}$ be given by $\phi\left(e^{1 \theta}\right)=a e^{1 \theta}+b e^{-1 \theta},|a|=|b|=\frac{1}{2}$ and $\rho(n)=n^{B}$. Then,

(i) foф is in $\mathrm{L}^{2}$ for all $f$ in $\mathrm{H}^{2}(\rho(n))$ if $\beta>\frac{1}{2}$,

(11) for each $B<\frac{1}{2}$ there is a function $f_{B}$ in $H^{2}(p(n))$ such that $f_{\beta}$ ob is not in $\mathrm{L}^{2}$

PROOF. As in the previous theorem we assume $a=b=\frac{1}{2}$ so that $f\left(\phi\left(e^{1 \theta}\right)\right)=f(\cos \theta)$. Let $f$ in $H^{2}(\rho)$ be given by $f(z)=\sum_{n=0}^{\infty} a_{n} z^{n}$. We have

$$
\left|f\left(\phi\left(e^{1 \theta}\right)\right)\right|^{2}=\left.||_{n=0}^{\infty} a_{n} \cos ^{n} \theta\right|^{2} \leq\left(\sum_{n=0}^{\infty}\left|a_{n}\right|^{2} \rho(n)\right)\left(\sum_{n=0}^{\infty} \frac{\cos ^{2 n} \theta}{\rho(n)}\right) .
$$

Now using (4.5) and $(4.6)$ as in the previous theorem it can be shown that

$$
\sin ^{2 \beta-2} \theta \sim \sum_{n=0}^{\infty} \frac{n^{-\beta}}{\Gamma(-\beta+1)} \cos ^{2 n} \theta \text {. }
$$

Thus fod is in $L^{2}$ if $\beta>\frac{1}{2}$.

For the proof of (i1) consider the function

$$
f(z)=\frac{1}{(1-z)^{\alpha+1}}=\sum A_{n}^{\alpha} z^{n} \text {. }
$$

By $(4.6)$,

$$
\sum\left|A_{n}^{\alpha}\right|^{2} n^{\beta} \sim \sum n^{2 \alpha+\beta}
$$

The sum on the right hand converges if $2 \alpha+\beta<-1$ i.e. $\alpha<-(\beta+1) / 2$. Thus, f is in $H^{2}(\rho), \rho(n)=n^{\beta}$ for $\alpha<-(\beta+1) / 2$. However,

$$
\int_{0}^{2 \pi}\left|f\left(\phi\left(e^{i \theta}\right)\right)\right|^{2} d \theta=\frac{1}{2^{2 \alpha+1}} \int_{0}^{\pi} \frac{1}{\sin ^{4 \alpha+4} \theta} d \theta=\infty
$$

if $\alpha \geq-\frac{3}{4}$. Thus, for given $\beta<\frac{1}{2}$, if we chose $\alpha=-(3+2 \beta+2) / 8$, $f$ is in $H^{2}(\rho)$ but foф is not in $L^{2}$.

REMARK. The case $\rho(n)=n^{\frac{1}{2}}$, remains open in the above theorem. However, in the next theorem we prove the same result for a sequence $p(n)$ having faster rate of growth than $n^{1 / 2}$ but with slower rate than $n^{1 / 2+\varepsilon}$ for any $\varepsilon>0$.

THEOREM 6. Let $\phi: T \rightarrow \bar{D}$ be as in the previous theorem and $p(n)=n^{1 / 2}(\log n)^{\beta}$. Then, (i) fop is in $\mathrm{L}^{2}$ for all $f$ in $\mathrm{H}^{2}(\rho(n))$ if $B>1$,

(1i) for each $\beta<0$, there is a function $f_{\beta}$ in $H^{2}\left(p(n)\right.$ ) such that $f_{B}$ o申 is not in $\mathrm{L}^{2}$. 
PROOF (1) Let $f$, given by $f(z)=\sum_{n=0}^{\infty} a_{n} z^{n}$, be in $H^{2}(p(n))$ so that

$$
\|f\|_{\rho}=\sum n^{1 / 2}(\log n)^{\beta}\left|a_{n}\right|^{2}<\infty .
$$

By Cauchy Schwarz inequality,

$$
\left.\left.\left|f\left(\phi\left(e^{1 \theta}\right)\right)\right|^{2} \leq n^{\infty}=0 n^{1 / 2}(\log n)^{\beta}\left|a_{n}\right|^{2}\right)\left(\sum_{n=0}^{\infty} \frac{\cos ^{2 n} \theta}{n^{1 / 2}(\log n)^{\beta}}\right)\right)
$$

It is known [7, p. 192] that if

$$
\frac{1}{(1-z)^{\alpha+1}}\left(\log \frac{a}{1-z}\right)^{\beta}={ }_{n=0}^{\infty} A_{n}^{(\alpha, \beta)} z^{n}
$$

then for $a>2, \alpha \neq-1,-2,-3, \ldots \ldots, \alpha, \beta \in R$

$$
A_{n}^{(\alpha, \beta)} \sim \frac{n^{\alpha}}{\Gamma(\alpha+1)}(\log n)^{\beta}
$$

so that

$$
A_{n}(-1 / 2,-\beta) \sim \frac{1}{\sqrt{\pi} n^{1 / 2}(\log n)^{\beta}}
$$

1.e.

$$
\frac{1}{\sqrt{\pi}} \sum_{n=0}^{\infty} \frac{\left(\cos ^{2} \theta\right)^{n}}{n^{1 / 2}(\log n)^{\beta}} \sim \frac{1}{\left(1-\cos ^{2} \theta\right)^{1 / 2}}\left(\log \frac{a}{1-\cos ^{2} \theta}\right)^{-\beta}=\frac{2^{-\beta}}{\sin \theta}\left(\log \frac{b}{\sin \theta}\right)^{-\beta},
$$

$\mathrm{b}=\sqrt{\mathrm{a}}$

In view of (4.7) and (4.9), In order to show that fod is in $L^{2}$, it is sufficient to show that

$$
\int_{0}^{2 \pi} \frac{1}{\sin \theta}\left(\log \frac{1}{\sin \theta}\right)^{-\beta} d \theta<\infty \quad .
$$

Further, because of the inequality $(2 \theta / \pi)<\sin \theta<\theta$, it is sufficlent to show the integrability, in an interval $(0, \delta)$, of the function

$$
h(\theta)=\frac{1}{\theta}\left(\log \frac{1}{\theta}\right)^{-\beta}
$$

Making the substitution $\log \left(\frac{1}{\theta}\right)=u$, we get

$$
\lim _{\varepsilon \rightarrow 0} \int_{\varepsilon}^{\delta} \frac{1}{\theta}\left(\log \frac{1}{\theta}\right)^{-\beta} d \theta=\lim _{\varepsilon \rightarrow 0} \frac{\left(\log \frac{1}{\varepsilon}\right)^{1-\beta}-\left(\log \frac{1}{\delta}\right)^{1-\beta}}{1-\beta}
$$

Thus, the above integral converges if $\beta>1$. This completes the proof of (1).

(11) Let $\rho(n)=n^{1 / 2}(\log n)^{-\beta}, \beta>0$. Now consider the function

$$
f(z)=\frac{1}{(1-z)^{1 / 4}}\left(\log \frac{a}{1-z}\right)^{\gamma}=\sum A_{n}^{\gamma} z^{n}
$$

where $-1 / 2>\gamma<(\beta-1) / 2$. We first observe that $f$ is in $H^{2}(\rho(n))$. In fact, a comparison of $f$ with (4.8) shows that

$$
A_{n}^{\gamma} \sim \frac{n^{-3 / 4}}{\Gamma(1 / 4)}(\log n)^{\gamma} \text {. }
$$

Thus,

$$
\Sigma\left|A_{n}^{\gamma}\right|^{2} \rho(n) \sim \Sigma n^{-1}(\log n)^{2 \gamma-\beta}
$$

and the right hand side series converges because $\gamma<(\beta-1) / 2$. Now, 


$$
\int_{0}^{2 \pi}\left|f\left(\phi\left(e^{1 \theta}\right)\right)\right|^{2} d \theta=2^{\gamma-1 / 2} 4 \int_{0}^{\pi / 2} \frac{1}{\sin \theta}\left(\log \frac{b}{\sin \theta}\right)^{2 \gamma} d \theta
$$

where $b=\frac{a}{2}$. The above integral diverges with the integral

$$
\int_{0}^{\pi / 2} \frac{1}{\theta}\left(\log \frac{1}{\theta}\right)^{2 \gamma} \mathrm{d} \theta
$$

because of the condition $\gamma>\frac{1}{2}$. Thus, we prove that although $f \varepsilon H^{2}(p(n))$, fo申 is not in $\mathrm{L}^{2}$.

REMARK. The case $\rho(n)=n^{\frac{1}{2}}(\log n)^{\beta}, 0 \leq \beta<1$ remains unsettled.

We conclude this section by showing that $P_{\phi}$ is an unbounded operator on $H^{2}$ for $\phi\left(e^{i t}\right)=\left(e^{i t}+e^{-i t}\right) / 2=\cos t$. This we do by exhibiting a sequence of function $f_{n}$ in $\mathrm{H}^{2}$ for which $\lim _{\mathrm{n} \rightarrow \infty}\left\|\mathrm{P}_{\phi} \mathrm{f}_{\mathrm{n}}\right\|=\infty$

Let $f_{n}(z)=\sum_{k=1}^{n} \cdot \frac{z^{k}}{k}$ and $f(z)=\sum_{k=1}^{\infty} \frac{z^{k}}{k}=\log \frac{1}{1-z}$ so that

$$
g_{n}(t)=f_{n}\left(\phi\left(e^{i t}\right)\right)=\sum_{k=1}^{n} \frac{\cos ^{k} t}{k} \text { and } g(t)=f\left(\phi\left(e^{i t}\right)\right)=\sum_{k=1}^{\infty} \frac{\cos ^{k} t}{k} \text {. }
$$

Observe that $g$ is in $L^{1}$ but is not in $L^{2}$. Let $a_{k}$ and $a_{n}^{(n)}$ respectively be the $k^{\text {th }}$ Fourier coefficients of $g$ and $g_{n}$. Since

$$
\lim _{n \rightarrow \infty} \int_{0}^{2 \pi} \lg _{n}(t)-g(t) \mid d t=0
$$

we get

$$
\begin{aligned}
& \lim _{n \rightarrow \infty} a_{n}^{(n)}=a_{k} \text {. Now, } \\
& \left.\lim _{n \rightarrow \infty}|| P_{\phi}\right|_{2} ^{2}=\lim _{n \rightarrow \infty} \sum_{k=-n}^{n}\left|a_{k}^{(n)}\right|^{2}=\sum_{k=-\infty}^{\infty}\left|a_{k}\right|^{2} \quad \frac{1}{2 \pi} s\left|g\left(e^{1 t}\right)\right|^{2} d t=\infty .
\end{aligned}
$$

5. COMPACTNESS OF $P_{\phi}$

In this section we discuss some examples 11 lustrating cases when $P_{\phi} 1 s$ compact and when it is not. Let $\phi_{1}, \phi_{2}, \phi_{3}: T \rightarrow \bar{D}$, be defined by
(1) $\phi_{1}\left(e^{1 t}\right)=a e^{-i t}$
$|a|=1$
$0 \leq t \leq 2 \pi$
(ii) $\phi_{2}\left(e^{i t}\right)\left[\begin{array}{l}e^{i t} \\ 0\end{array}\right.$
$0 \leq t<\pi$
(1ii) $\phi_{3}\left(e^{i t}\right)=\left[\begin{array}{l}e^{1 t} \\ a e^{1 t}+b e^{-1 t}\end{array}\right.$,
$\pi \leq t \leq 2 \pi$
$0 \leq t \leq \pi$

(i) $P_{\phi 1}$ is a finite rank, hence a compact, operator. For, if $f$ in $H^{2}$ is given by $f(z)=\sum_{n=0}^{\infty} a_{n} z^{n}$ then $\left(P_{\phi_{1}} f\right)\left(e^{1 t}\right)=P\left(\sum_{n=0}^{\infty} a_{n} a^{n} e^{-1 n t}\right)=a_{0}$. 
For composition operators with analytic $\phi$ Schwartz [8] has shown that if $C_{\phi}:{ }_{H}^{\mathrm{P}}(D) \rightarrow{ }_{H}^{\mathrm{P}}(D)$ is compact then $\left|\phi\left(e^{1 t}\right)\right|<1$ a.e. where $\phi\left(e^{1 t}\right)$ is the radial limit of $\phi(z)$. We observe in this example that $P_{\phi 1}$ deviates in behaviour from $C_{\phi}$. (11) We have shown at the end of section 3 that $\left\|P_{\phi_{2}}\right\|<\sqrt{2}$. We show here that $P_{\phi_{2}}$ is not compact.

By Riemann-Lebesgue Lemma the sequence $e_{n}, n=0,1,2, \ldots$ converges to zero weakly in $\mathrm{H}^{2}$. However, $\mathrm{P}_{\phi_{2}}\left(e_{\mathrm{n}}\right)=P\left(e_{n} \circ \phi_{2}\right)$, does not converge strongly to zero. For, if the Fourier series of $e_{n} 0 \phi_{2}$ is given by $\left(e_{n} \circ \phi_{2}\right)\left(e^{1 t}\right)=\sum_{m=-\infty}^{\infty} a_{m} e^{1 m t}$, then by direct computation it can be seen that

$$
a_{m}=\left[\begin{array}{lll}
\frac{1}{\pi(n-m)} & \text { if } n-m \text { is odd } \\
0 & \text { if } n-m \text { is even } \\
\frac{1}{2} & \text { if } n=m
\end{array}\right.
$$

and $\left\|P_{\phi_{2}}\left(e_{n}\right)\right\|_{2}^{2}=\sum_{m=0}^{\infty}\left|a_{m}\right|^{2}>\frac{1}{4}$. Thus, $P_{\phi_{2}}$ is not compact.

By a similar argument as in (11) it can be shown that $P_{\phi_{3}}$ is bounded but not a compact operator.

ACKNOWLEDGEMENT. The authors thank the referee for his useful suggestions regarding the defintion of $P_{\phi}$.

\section{REFERENCES}

1. DUREN, P.L., $\mathrm{H}^{\mathrm{p}}$ Spaces, Academic Press, New York, 1970.

2. HOFFMAN, K., Banach Spaces of Analyt1c Functions, Prentic-Ha11, Englewood C1iffs, New Jersey, 1962.

3. HALMOS, P.R., A Hilbert Space Problem Book, Springer-Verlag, New York, Inc., Gra duate texts in Mathematics, Vol. 19.

4. NORDGREN, E.A., Composition Operators in H1lbert Spaces, Lecture notes in Mathematics, 639, Springer-Verlag, Berlin, 1978.

5. CARLESON, L., On Convergence Growth of Partial Sums of Fourier Series. Acta Math, $116(1966), 135-157$.

6. GABRIEL, R.M., Some Results Concerning the Moduli of Regular Functions Along Curves of Certain Types, Proc. London Math. Soc. 28(1928), 121-127.

7. ZYGMOND, A., Trigonometric Ser1es, 2nd Ed., Cambridge University Press, 1959.

8. SCHWARTZ, H. Composition Operators on H, Dissertation, University of Toledo, 1969 


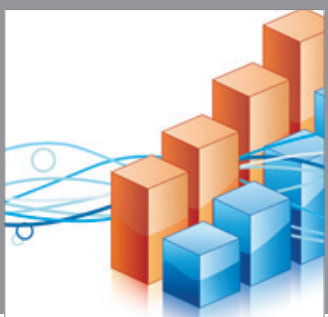

Advances in

Operations Research

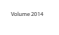

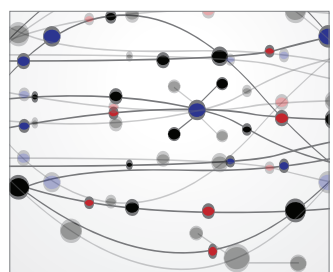

\section{The Scientific} World Journal
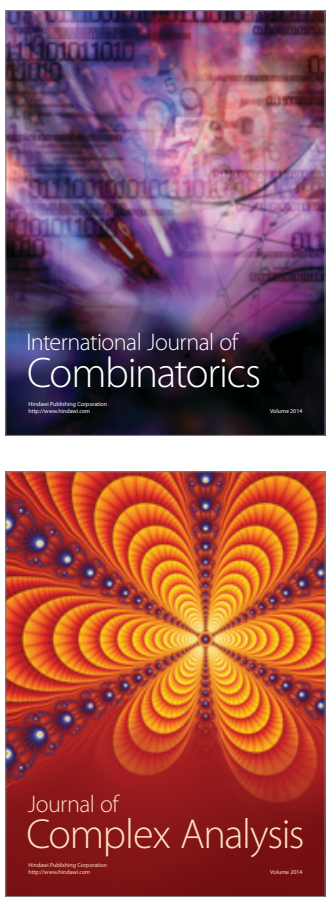

International Journal of

Mathematics and

Mathematical

Sciences
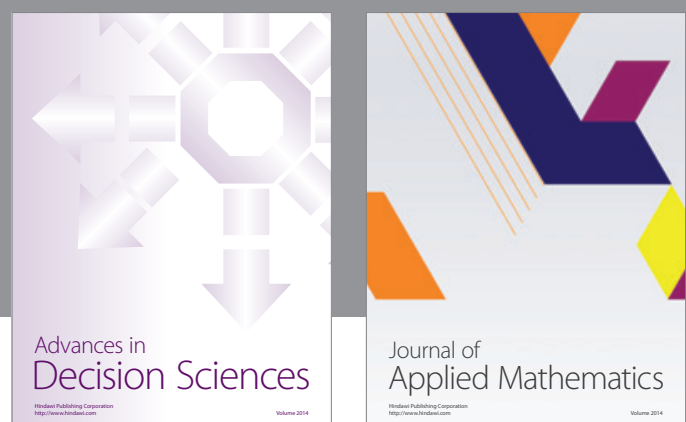

Journal of

Applied Mathematics
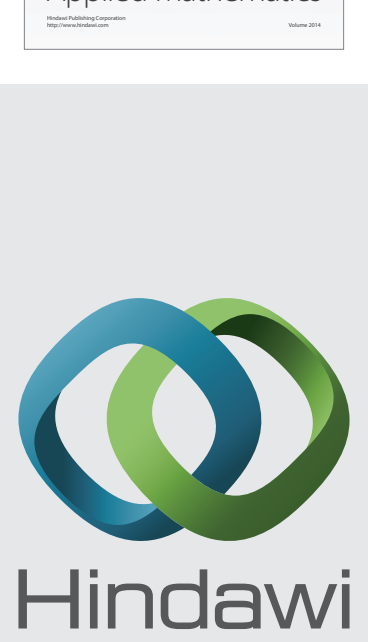

Submit your manuscripts at http://www.hindawi.com
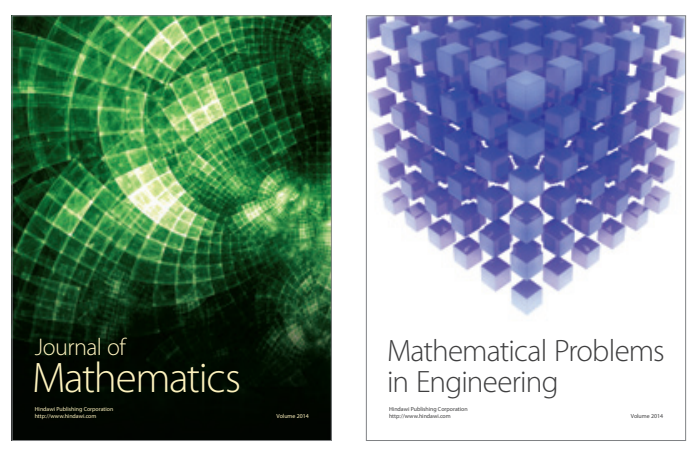

Mathematical Problems in Engineering
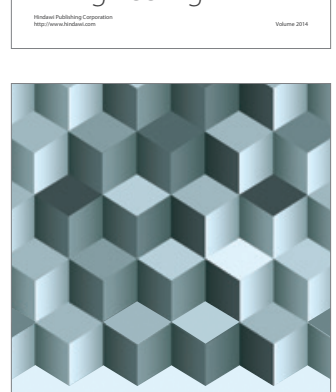

Journal of

Function Spaces
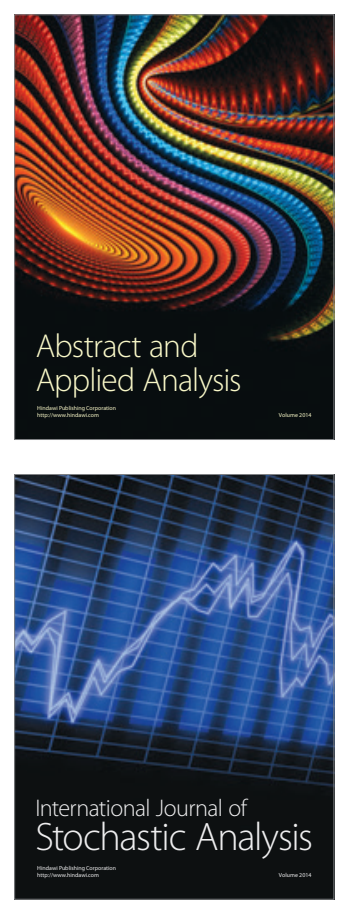

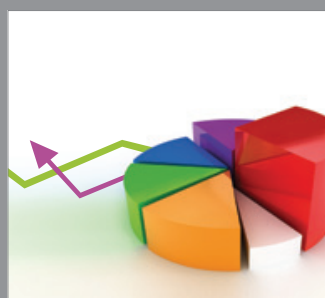

ournal of

Probability and Statistics

Promensencen
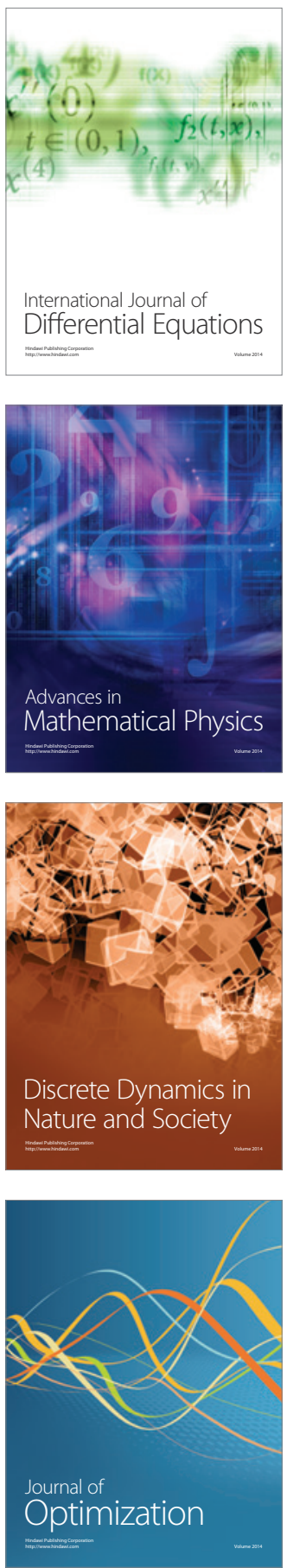